\title{
SOBOLEV EMBEDDINGS IN METRIC MEASURE SPACES WITH VARIABLE DIMENSION
}

\author{
PETTERI HARJULEHTO, PETER HÄSTÖ* AND VISA LATVALA
}

\begin{abstract}
In this article we study metric measure spaces with variable dimension. We consider Lebesgue spaces on these sets, and embeddings of the Riesz potential in these spaces. We also investigate Hajłasz-type Sobolev spaces, and prove Sobolev and Trudinger inequalities with optimal exponents. All of these questions lead naturally to function spaces with variable exponents.
\end{abstract}

\section{INTRODUCTION}

Fractal-type spaces have been considered in connection with a vast variety of phenomena since the late 1970's. Early work was concerned with estimating the fractal dimension of various things, and deriving some first order estimates based on this, see e.g. [10, 29]. More recently, there has been an interest in doing analysis on fractal-type spaces, with the eventual hope of deriving more detailed information about the processes (described e.g. by PDEs) taking place in the spaces, see e.g. [13, 14, 21]. However, also in the latter investigations, the dimension has been treated as a single number, typically a vast overestimate derived from a doubling condition. We will argue that the space can be better described by a variable dimension.

One recent mathematical investigation coming very close to a variable dimension is the study by Hambly and Kumgai [16]: they study differential equations on spaces where a countable number of fractal sets with different dimensions are embedded into $\mathbb{R}^{2}$. It is fair to ask whether it would not be simpler to replace this by a continuum of fractals with continuously varying dimension.

Let us also consider a physical example as motivation for variable dimensional spaces: take the case of the surface of a silica gel. As described in $[2,9,29]$ the dimension of this surface is close to 3 , and, moreover, it varies both with the exact type of material and with the manufacturing process. Thus we can easily conceive of a situation where there is, say, a gradient of decreasing surface dimension.

Date: August 31, 2005.

2000 Mathematics Subject Classification. Primary 46E35; Secondary 28A78, 28A80, 42B20, 46E30.

Key words and phrases. Variable exponent, variable dimension, Hausdorff measure, Lebesgue space, Riesz potential, Hajłasz space, metric measure space.

* Supported the Research Council of Norway, Project 160192/V30. 
These kind of spaces, with variable dimension, are the object of this study. In order to consider differential equations in the space, the first step is to define a Lebesgue space on the set. After this, we can define spaces of differentiable functions: in this article we will use the definition due to Hajłasz [13]. Moreover, to arrive at such classical tools as the Sobolev inequality in this setting automatically involves a variable exponent version of the Lebesgue space. Many fundamental tools of classical theory have been worked out recently in the variable exponent, Euclidean setting, see e.g. $[1,3,6,17,24,25]$, and some studies in the metric space setting have also appeared [12, 18, 19, 20, 27].

In this article we expand the metric measure spaces framework by studying Lebesgue spaces on fractal sets with variable dimension. It turns out that many results can be directly transferred to this setting once we have chosen an appropriate regularity condition - lower Ahlfors regular spaces. Notice that the commonly used doubling assumption is stronger that lower Ahlfors regularity, but weaker than Ahlfors regularity. In Section 3 we give precise definitions of what we mean by a variable dimension Hausdorff measure and, as an example, define a corresponding von Koch curve. In Section 4 we show that the appropriate Riesz potential operator is bounded even for measures that are only lower Ahlfors regular. In Section 5 we prove the TrudingerSobolev inequality in the variable dimension case with point-wise optimal exponent. All our embedding results are improved versions of the standard results in the sense that they take into account the local variable dimension.

Notation. By a metric measure space we mean a triple $(X, d, \mu)$, where $X$ is a set, $d$ is a metric on $X$ and $\mu$ is a non-negative, Borel regular outer measure on $X$, which is finite in every bounded set. For simplicity, we often write $X$ instead of $(X, d, \mu)$. For $x \in X$ and $r>0$ we denote by $B(x, r)$ the open ball centered at $x$ with radius $r ; \bar{B}(x, r)$ denotes the corresponding closed ball. We use the convention that $C$ denotes a generic constant, i.e. a constant whose values can change even between different occurrences in a chain of inequalities. The notation $u \lesssim v$ means that there exists a constant $C$ such that $u \leq C v$, and $u \approx v$ means that $u \lesssim v$ and $v \lesssim u$.

Variable exponent spaces. By a variable exponent we mean a bounded measurable function $p: X \rightarrow[1, \infty)$. For $A \subset X$ we set $p_{A}^{+}=\operatorname{ess}_{\sup _{x \in A}} p(x)$ and $p_{A}^{-}=\operatorname{essinf}_{x \in A} p(x)$; we abbreviate $p^{+}=p_{X}^{+}$and $p^{-}=p_{X}^{-}$. For a $\mu$-measurable function $u: X \rightarrow \mathbb{R}$ we define the modular

$$
\varrho_{p(\cdot)}(u)=\int_{X}|u(y)|^{p(y)} d \mu(y)
$$

and the norm

$$
\|u\|_{p(\cdot)}=\inf \left\{\lambda>0: \varrho_{p(\cdot)}(u / \lambda) \leqslant 1\right\} .
$$

The variable exponent Lebesgue space $L^{p(\cdot)}(X, d, \mu)$ consists of those $\mu$-measurable functions $u: X \rightarrow \mathbb{R}$ for which $\|u\|_{p(\cdot)}<\infty$. Some basic properties of variable exponent Lebesgue spaces on metric measure spaces are given in 
[19]. Most of these are straightforward generalizations of the Euclidean case [25]. In what follows, we will use the generalized Hölder inequality,

$$
\int_{X} f(x) g(x) d \mu(x) \leq 3\|f\|_{p(\cdot)}\|g\|_{p^{\prime}(\cdot)}
$$

[25, Theorem 2.1], and the elementary estimate

$$
\min \left\{\|f\|_{p(\cdot)}^{p^{+}},\|f\|_{p(\cdot)}^{p^{-}}\right\} \leq \varrho_{p(\cdot)}(u) \leq \max \left\{\|f\|_{p(\cdot)}^{p^{+}},\|f\|_{p(\cdot)}^{p^{-}}\right\}
$$

without any further reference.

We say that the function $p$ is log-Hölder continuous if

$$
|p(x)-p(y)| \leq \frac{C}{\log (1 / d(x, y))}
$$

for all points with $d(x, y)<\frac{1}{2}$. It has been shown that this condition is sufficient for proving many regularity results on variable exponent spaces. In particular, if $1<p^{-} \leq p^{+}<\infty$ and $p$ is log-Hölder continuous, then the maximal operator is locally bounded in $L^{p(\cdot)}[4,19]$.

\section{Measure Regularity COnditions}

In the context of metric measure spaces it is natural to assume some connection between the measure and the metric. What we mean by dimension is some quantity which relates the measure of a ball to its radius. Thus, if we are given a measure space, we can either try to choose the metric so that the dimension is constant, or choose the dimension so that the metric is in some way natural.

The former trick was used by Hambly in [15]. In that study, a Sierpinskigasket type fractal is constructed, in which the density parameter (which determines the dimension) is chosen randomly at each level of the construction. By defining a suitable metric, Hambly neatly avoids the complications of a variable dimension, however, in so doing, he cannot use the natural, inherited metric from $\mathbb{R}^{2}$. As we will show in this paper, very little regularity is really needed for the variable dimension in order to get the usual results in Lebesgue spaces. Thus one can ask whether modifying distances is really warranted?

Perhaps the best-known regularity assumption on measures in the general setting is Ahlfors regularity. We say that $\mu$ is Ahlfors $Q$-regular if

$$
\mu(B(x, r)) \approx r^{Q}
$$

for all $x \in X$ and $r \leq \operatorname{diam} X$. In this setting it is natural to call $Q$ the dimension of the metric space.

As was argued in the introduction, it is of interest to study also spaces with variable dimension. Thus, if $Q: X \rightarrow(0, \infty)$ is a BOUNDED function, then we say that $\mu$ is Ahlfors $Q(\cdot)$-regular if

$$
\mu(B(x, r)) \approx r^{Q(x)}
$$


for all $x \in X$ and $r \leq \operatorname{diam} X$. Ahlfors $Q(\cdot)$-regularity is only possible for sufficiently regular functions $Q$ :

Lemma 2.1. If $\mu$ is Ahlfors $Q(\cdot)$-regular, then $Q$ is log-Hölder continuous.

Proof. Choose $x, y \in X$ and assume $r=d(x, y)<1$. Then

$$
r^{Q(x)} \approx \mu(B(x, 2 r)) \geq \mu(B(y, r)) \approx r^{Q(y)},
$$

and similarly $r^{Q(x)} \lesssim r^{Q(y)}$. Hence

$$
d(x, y)^{Q(x)-Q(y)} \approx 1
$$

for all $x, y \in X$, which is equivalent to log-Hölder continuity.

Another well established condition in the context of metric measure spaces is the doubling condition. Recall that the measure $\mu$ is doubling if

$$
\mu(B(x, 2 r)) \leq C_{\mu} \mu(B(x, r))
$$

for every open ball $B(x, r)$ in $X$. The constant $C_{\mu}$ is called the doubling constant.

We also introduce a weaker condition: for a function $Q$ as before we say that $\mu$ is lower Ahlfors $Q(\cdot)$-regular if

$$
\mu(B(x, r)) \gtrsim r^{Q(x)}
$$

for all $x \in X$ and $r \in(0,1)$. If $Q$ is constant, we simply say that $\mu$ is lower Ahlfors $Q$-regular. For bounded spaces $X$, the condition can be equivalently assumed to hold for all $r \in(0, \operatorname{diam} X)$. It directly follows that a lower Ahlfors $Q(\cdot)$-regular measure is lower Ahlfors $Q^{+}$-regular (as before, $Q^{+}=$ $\left.\sup _{x \in X} Q(x)\right)$ so in this case our notion is easily related to existing theory.

It is immediately clear that every Ahlfors $Q(\cdot)$-regular measure is doubling. Suppose next that $X$ is a bounded space with doubling measure. If $B(y, R)$ is an open ball in $X, x \in B(y, R)$ and $0<r \leq R<\infty$, then

$$
\frac{\mu(B(x, r))}{\mu(B(y, R))} \gtrsim\left(\frac{r}{R}\right)^{Q_{\mu}}
$$

for $Q_{\mu}=\log _{2} C_{\mu}$. Choosing $R=\operatorname{diam} X$ we see that the doubling condition implies lower Ahlfors regularity.

Lower Ahlfors regularity and log-Hölder continuity of the exponent $p$ together imply certain very useful estimates on the measures of balls:

Lemma 2.2 (Lemma 3.6, [19]). Let $\mu$ be lower Ahlfors $Q$-regular and let $p$ be log-Hölder continuous. Then there is a constant $K$ such that

$$
\mu(B)^{p_{B}^{-}-p_{B}^{+}} \leq K
$$

for all balls $B \subset X$. 
Lemma 2.3. Let $\mu$ be lower Ahlfors $Q$-regular in a bounded set $X$ and let $p$ be log-Hölder continuous and bounded in $X$. Then for every ball $B \subset X$ centered at $x$ we have

$$
\int_{B} \mu(B)^{\frac{-p(y)}{p(x)}} d \mu(y) \leq \max \left\{\mu(X)^{\frac{p^{+}}{p^{-}}}, K^{\frac{1}{p^{-}}}\right\},
$$

where $K \geq 1$ is the constant of Lemma 2.2.

Proof. By the previous lemma we know that $\mu(B)^{p_{B}^{-}-p_{B}^{+}} \leq K$ for all balls $B \subset X$. Thus

$$
\mu(B)^{p(x)-p(y)} \leq \max \left\{\mu(B)^{p_{B}^{+}-p_{B}^{-}}, \mu(B)^{p_{B}^{-}-p_{B}^{+}}\right\} \leq \max \left\{\mu(B)^{p^{+}}, K\right\}
$$

for every $x, y \in B$. Hence we have

$$
\begin{aligned}
\int_{B(x, r)} \mu(B(x, r))^{\frac{-p(y)}{p(x)}} d \mu(y) & =\int_{B(x, r)} \mu(B(x, r))^{\frac{p(x)-p(y)}{p(x)}} d \mu(y) \\
& \leq \max \left\{\mu(B)^{p^{+}}, K\right\}^{\frac{1}{p^{-}}} f_{B(x, r)} d \mu(y),
\end{aligned}
$$

which completes the proof.

The following fact is proven like the previous lemma.

Lemma 2.4. Let $\mu$ be lower Ahlfors $Q$-regular in a bounded set $X$ and let $p$ be bounded and log-Hölder continuous. Then for every ball $B$ containing $x$

$$
\|1\|_{L^{p(\cdot)}(B)} \leq C \mu(B)^{\frac{1}{p(x)}} .
$$

In the rest of this paper we will prove several result for variable dimension metric measure spaces. The following trivial proposition guarantees that these results are always at least as good as the constant-dimension results that have been proven by other researchers.

Proposition 2.5. Let $X$ be a metric measure space with a doubling measure $\mu$. Suppose also that the space is lower $Q(\cdot)$-Ahlfors regular. Then $X$ is lower $\tilde{Q}(\cdot)$-Ahlfors regular, where $\tilde{Q}(x)=\max \left\{Q(x), Q_{\mu}\right\}$.

\section{MeAsures of VARIABle Dimension}

There is a standard way to relate measures and distances in metric measure spaces to give some kind of dimension. The following definition of point-wise upper and lower local dimensions appear e.g. in [7, 8, 22]:

and

$$
\overline{\operatorname{dim}} X(x)=\limsup _{r \rightarrow 0} \frac{\log \mu(B(x, r))}{\log r}
$$

$$
\underline{\operatorname{dim}} X(x)=\liminf _{r \rightarrow 0} \frac{\log \mu(B(x, r))}{\log r},
$$

where $X \subset \mathbb{R}^{n}$. Measures for which $\underline{\operatorname{dim}} X(x)=\overline{\operatorname{dim}} X(x)=C$ almost everywhere are called exact dimensional in [7], and have been studied to some extent. The strategy in [7] for studying non-exact (multifractal) measures is 
to consider them as composed of infinitely many exact measures. This is in some sense analogous to thinking of a function as a sum of simple functions. As this analogy suggests, it may be worthwhile to look at the measures also from a more global point-of-view - in this case the dimension is described by a function varying with the point $x$. With this perspective, we will define a variable dimension Hausdorff measure which is very far from exact, and show that it is the appropriate measure for a certain von Koch-type curve.

Hausdorff measures. The definition of the variable Hausdorff measure is fairly straightforward. Let $Q: X \rightarrow(0, \infty)$ be a bounded function. We define the variable Hausdorff measure by first letting

$$
\mathcal{H}_{\delta}^{Q(\cdot)}(E)=\inf \left\{\sum_{i} \operatorname{diam}\left(B_{i}\right)^{Q\left(x_{i}\right)}: E \subset \bigcup_{i} B_{i}, \operatorname{diam}\left(B_{i}\right) \leq \delta\right\},
$$

where $x_{i}$ is the center of $B_{i}$, and then taking the limit:

$$
\mathcal{H}^{Q(\cdot)}(E)=\lim _{\delta \rightarrow 0} \mathcal{H}_{\delta}^{Q(\cdot)}(E)
$$

This is just a special case of a measure construction due to Carathéodory, hence $\mathcal{H}^{Q(\cdot)}$ is a Borel regular outer measure [11, 2.10.1, p. 169].

Note that there is some degree of arbitrariness in choosing the value of $s$ at the center of the ball. We can similarly define lower and upper variable Hausdorff measures by taking the limit $\delta \rightarrow 0$ of

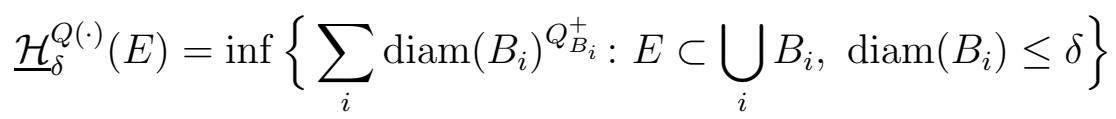

and

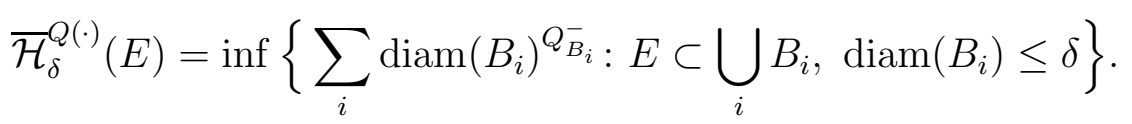

However, we have the following uniqueness result:

Proposition 3.1. If $Q: X \rightarrow(0, \infty)$ is log-Hölder continuous, then

$$
\overline{\mathcal{H}}^{Q(\cdot)}(E) \approx \underline{\mathcal{H}}^{Q(\cdot)}(E) \approx \mathcal{H}^{Q(\cdot)}(E)
$$

for every $E \subset X$.

Proof. For any $x \in X$ and $r<1 / 2$ we have

$$
1 \leq \operatorname{diam}(B(x, r))^{Q_{B(x, r)}^{-}-Q_{B(x, r)}^{+}} \leq C
$$

by the log-Hölder continuity condition, so the claim follows.

Von Koch curves. We next construct a variable dimensional von Koch curve, modifying the standard procedure which can be found in any basic book of modern real analysis or measure theory, see e.g. [26].

We construct a sequence of piece-wise linear curves. The length of the segment $K$ will be denoted by $|K|$. Let a function $s:[0,1] \rightarrow(1 / 4,1 / 2)$ be given. We start with the unit segment $[0,1] \subset \mathbb{R}^{2}$. We divide this into four equally long segments, and replace each subsegment with a segment of 


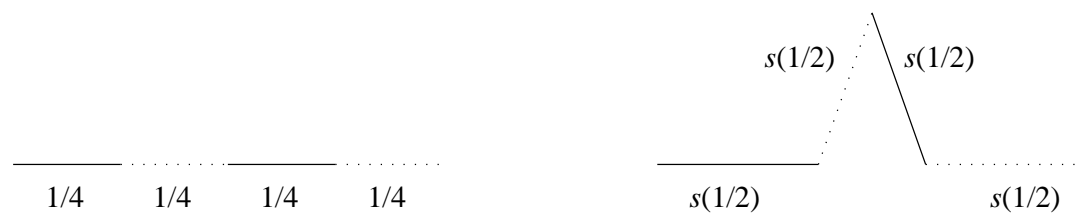

Figure 1. The construction of the von Koch curve.
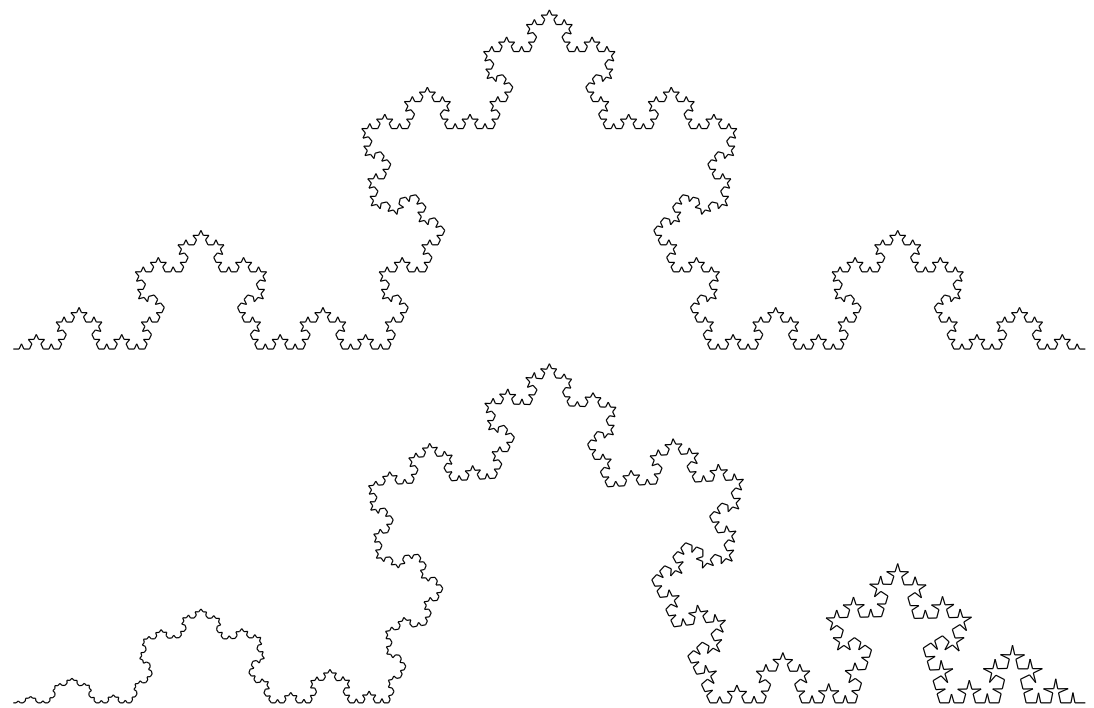

Figure 2. An ordinary Koch curve (upper) and a variable dimension Koch curve (lower).

length $s(1 / 2)|K|$, in such a way that the segments $K_{0}$ and $K_{3}$ are subsets of $K$ at opposite ends of $K$, and $K_{1}$ and $K_{2}$ are the sides of an isosceles triangle whose base is $K \backslash\left(K_{0} \cup K_{3}\right)$, see Figure 1. Notice that the right curve in the figure is naturally parameterized by the unit interval; we denote by $\Phi_{(1)}$ this map of the unit interval onto the first level of the construction.

We repeat this process in each of the four segments, but using the lengths give by the $s\left(x_{i}\right)$-value, where $x_{i}$ is the parameter value of the middle point of the segment, so the first four segments $\left(K_{00}, K_{01}, K_{02}, K_{03}\right)$ have lengths $s(1 / 8)\left|K_{0}\right|$, the next four $s(3 / 8)\left|K_{1}\right|$, then $s(5 / 8)\left|K_{2}\right|$, and the last four $s(7 / 8)\left|K_{3}\right|$. In general, we denote by $K_{i_{1} \ldots i_{k}}$ the segments from the $k^{\text {th }}$ level of the construction, where every $i_{j}$ belongs to $\{0,1,2,3\}$. The corresponding parameter map is denoted by $\Phi_{(k)}$. This construction is continued, and the von Koch curve is the limit set. Of course, if $s$ equals a constant, we get the ordinary von Koch curve. The fifth level of this construction is illustrated in Figure 2 for $s \equiv 0.35$ and $s(t)=0.25+t / 5$. The construction also directly produces a continuous map $\Phi$ from $[0,1]$ to the von Koch curve.

We want to show that if the function $s$ is reasonably regular, then the construction produces a curve with variable dimension given by

$$
Q(x)=\frac{\log 4}{\log (1 / s(x))} .
$$


Of course, if $s$ is a constant function, then this is well-known. We will use the following lemma.

Lemma 3.3. Let $s:[0,1] \rightarrow(1 / 4,1 / 2)$ be Lipschitz and let $K_{s(\cdot)}$ be the corresponding von Koch curve. Then

$$
\left|K_{i_{1} \ldots i_{k}}\right| \approx s(x)^{k}
$$

for every $x \in \Phi_{(k)}^{-1}\left(K_{i_{1} \ldots i_{k}}\right)$, where the implicit constants are independent of $k$.

Proof. Recursively we find that

$$
\left|K_{i_{1} \ldots i_{k}}\right|=\left|K_{i_{1} \ldots i_{k-1}}\right| s\left(x_{k}\right)=\ldots=|K| s\left(\frac{1}{2}\right) s\left(\frac{2 x_{1}-1}{8}\right) \cdots s\left(\frac{2 x_{k}-1}{2 \cdot 4^{k}}\right),
$$

where $x_{j} \in\left[1,4^{j}\right]$ is an integer chosen so that $\frac{2 x_{j}-1}{2 \cdot 4^{j}}$ is as close to $x$ as possible. This choice of $x_{j}$ implies that

$$
\left|\frac{2 x_{j}-1}{2 \cdot 4^{j}}-x\right| \leq \frac{1}{2 \cdot 4^{j}} .
$$

Let $L>0$ denote the Lipschitz constant of $s$. Then

$$
\begin{aligned}
& s\left(\frac{1}{2}\right) s\left(\frac{2 x_{1}-1}{8}\right) \cdots s\left(\frac{2 x_{k}-1}{2 \cdot 4^{k}}\right) \leq\left(s(x)+\frac{L}{2}\right)\left(s(x)+\frac{L}{8}\right) \cdots\left(s(x)+\frac{L}{2 \cdot 4^{k}}\right) \\
& \quad \leq\left(1+\frac{L}{2 s^{-}}\right)\left(1+\frac{L}{8 s^{-}}\right) \cdots\left(1+\frac{L}{2 \cdot 4^{k} s^{-}}\right) s^{+} s(x)^{k} \\
& \quad \leq C_{K} s(x)^{k},
\end{aligned}
$$

where $C_{K}=\exp \left(L / s^{-}\right) s^{+}$and the last estimate is derived using the inequality $1+z \leq \exp z$ for each of the terms. Similarly, we derive the lower bound $s(x)^{k} / C_{K}$ for the product.

We next show that the variable dimensional Hausdorff measure is indeed suitable for our Koch curve. The idea with the proof is that the Hausdorff measure of a ball $B \subset K_{s(\cdot)}$ is essentially the Euclidean measure of the preimage $\Phi^{-1}(B) \subset[0,1]$.

Theorem 3.4. Let $s:[0,1] \rightarrow(1 / 4,1 / 2)$ be Lipschitz, let $K_{s(\cdot)}$ be the corresponding von Koch curve and let $Q$ be as in (3.2). Then

$$
0<\mathcal{H}^{Q(\cdot)}\left(K_{s(\cdot)}\right)<\infty
$$

and $\mathcal{H}^{Q(\cdot)}$ is Ahlfors $Q(\cdot)$-regular.

Proof. For a segment $K_{i_{1} \ldots i_{k}}$ in the construction of the von Koch curve we denote by $B\left(K_{i_{1} \ldots i_{k}}\right)$ the open ball whose diametrical chord is $K_{i_{1} \ldots i_{k}}$. We define ball families by

$$
\mathcal{B}_{k}=\left\{B\left(K_{i_{1} \ldots i_{k}}\right): i_{j} \in\{0,1,2,3\}\right\} .
$$

The curve $K_{s(\cdot)}$ is covered by the $4^{k}$ balls in $\mathcal{B}_{k}$ (except for a finite number of points, which clearly constitute a set of measure zero). Denoting $\delta=$ $\max \left|K_{i_{1} \ldots i_{k}}\right|$, we infer using Lemma 3.3 that

$$
\mathcal{H}_{\delta}^{Q(\cdot)}\left(K_{s(\cdot)}\right) \leq C_{K} \sum_{i=1}^{4^{k}} s\left(x_{i}\right)^{k Q\left(x_{i}\right)}=C_{K} \sum_{i=1}^{4^{k}} 4^{-k}=C_{K},
$$


where $x_{i}=\frac{2 i-1}{2 \cdot 4^{k}}$ and we used the assumption $Q(x)=\frac{\log 4}{\log (1 / s(x))}$ for the secondto-last equality. Since $\delta$ tends to zero as $k \rightarrow \infty$, this implies that

$$
\mathcal{H}^{Q(\cdot)}\left(K_{s(\cdot)}\right) \leq C_{K}
$$

Next we derive a lower bound for $\mathcal{H}_{\delta}^{Q(\cdot)}\left(K_{s(\cdot)}\right)$. So let $\left(B_{i}\right)$ be a cover by balls of $K_{s(\cdot)}$. Since $K_{s(\cdot)}$ is compact we find a finite subcover $B_{i}=B\left(x_{i}^{\prime}, r_{i}\right)$ of $K_{s(\cdot)}$. For each ball $B_{i}$ we can find a part $K_{i}$ of the curve which is contained in $B_{i}$ and whose length is at least half of $r_{i}$. We denote $x_{i}=\Phi^{-1}\left(x_{i}^{\prime}\right)$. Then we have

$$
\sum_{i} \operatorname{diam}\left(B_{i}\right)^{Q\left(x_{i}\right)} \approx \sum_{i}\left|K_{i}\right|^{Q\left(x_{i}\right)} \approx \sum_{i} s\left(x_{i}\right)^{k_{i} Q\left(x_{i}\right)}=\sum_{i} 4^{-k_{i}} \geq \frac{1}{4},
$$

where $k_{i}$ denotes the level of $K_{i}$. The last inequality holds, since at least one quarter of every ball contributes to the sum, and the whole balls cover the unit interval.

Proving Ahlfors $Q(\cdot)$-regularity follows exactly the same line of argument, we just replace the set $K_{s(\cdot)}$ by the subset which is contained in the ball whose measure we are estimating.

Remark 3.5. The assumption that $s$ is Lipschitz in the previous theorem is necessary in the following sense: if the Hausdorff measure is assumed to be $Q(\cdot)$-Ahlfors regular, with $Q(x)=\frac{\log 4}{\log (1 / s(x))}$, then we easily conclude by Lemma 2.1 that $s$ is Lipschitz.

\section{Embeddings For Riesz potentials}

In this section we assume that $X$ is a bounded subset of $\mathbb{R}^{n}$ equipped with the standard Euclidean metric and a measure $\mu$, which is Borel regular and finite in every ball. Moreover, we assume that $\mu$ is lower Ahlfors $Q$-regular. The reason for focusing on the subsets of $\mathbb{R}^{n}$ is that we need the maximal function theorem, which is known, without a doubling condition, only for subsets of $\mathbb{R}^{n}$ (but see also Remark 4.3).

The Hardy-Littlewood maximal operator. For a locally integrable function $u$ on $X$, we define the Hardy-Littlewood maximal operator by

$$
\mathcal{M} u(x)=\sup _{r>0} f_{B(x, r)}|u| d \mu .
$$

Under our assumptions the Hardy-Littlewood maximal operator satisfies the following weak and strong type estimates. These are easy consequences of the standard results.

Lemma 4.1. Let $\mu$ be lower Ahlfors $Q$-regular in a bounded set $X \subset \mathbb{R}^{n}$. For all $t>0$ and for any constant exponent $p>1$ we have

$$
\mu(\{\mathcal{M} u>t\}) \leq \frac{C_{1}}{t} \int_{X}|u| d \mu
$$


and

$$
\int_{X}|\mathcal{M} u|^{p} d \mu \leq C_{2} \int_{X}|u|^{p} d \mu .
$$

Proof. We extend $\mu$ to the whole of $\mathbb{R}^{n}$ by setting $\mu(A)=\mu(A \cap X)$ for all $A \subset \mathbb{R}^{n}$. This extended $\mu$ is a Radon measure on $\mathbb{R}^{n}$, see [26, Corollary 1.11]. The claim then follows from [26, Theorem 2.19].

Combining Lemmas 4.1 and 2.2 with the arguments in [19, Lemma 4.2 and Theorem 4.3] yields that the Hardy-Littlewood maximal operator is bounded also in the variable exponent space under our assumptions.

Theorem 4.2. Let $\mu$ be lower Ahlfors $Q$-regular in a bounded set $X \subset \mathbb{R}^{n}$ and let $p$ be log-Hölder continuous in $X$ with $1<p^{-} \leq p^{+}<\infty$. Then

$$
\|\mathcal{M} u\|_{p(\cdot)} \leq C\|u\|_{p(\cdot)}
$$

for all $u \in L^{p(\cdot)}(X)$.

Remark 4.3. Instead of assuming that $X$ is a subset of $\mathbb{R}^{n}$ we could more generally assume that $X$ is directionally $(\varepsilon, M)$-limited in the sense of $[21$, p. 7].

Riesz-potentials. Let $\alpha>0$ be fixed. Following [14] we consider a Riesztype operator which is better suited to the metric measure space setting. We define for an open set $\Omega \subset X$ and an integrable function $u$ an operator

$$
J_{\alpha}^{\Omega} u(x)=\sum_{2^{i} \leq 2 \operatorname{diam} \Omega} 2^{i \alpha} f_{B\left(x, 2^{i}\right)}|u(y)| d \mu(y) .
$$

Here, and later, the sum should be understood to be taken over all integers $i$ satisfying $2^{i} \leq 2 \operatorname{diam} \Omega$. If the measure $\mu$ is doubling, then $I_{\alpha}^{\Omega}|u|(x) \leq$ $J_{\alpha}^{\Omega} u(x)$ for almost every $x \in X$, where

$$
I_{\alpha}^{\Omega}|u|(x)=\int_{\Omega} \frac{|u(y)| d(x, y)^{\alpha}}{\mu(B(x, d(x, y))} d \mu(y)
$$

is the usual Riesz potential. We abbreviate $J_{\alpha}=J_{\alpha}^{X}$. Riesz potentials in the variable exponent setting have to been studied for instance in $[5,12,23,28]$.

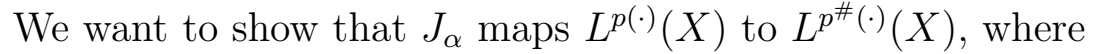

$$
p^{\#}(x)=\frac{Q(x) p(x)}{Q(x)-\alpha p(x)} .
$$

From the constant exponent case we know that we can only hope to achieve this when $Q(x)-\alpha p(x)$ is bounded away from 0 . The proof of the following result is based on [14, Theorem 5.3].

Proposition 4.4. Let $\mu$ be lower Ahlfors $Q(\cdot)$-regular in a bounded set $X \subset$ $\mathbb{R}^{n}$. Let $p$ be log-Hölder continuous in $X$ with $1<p^{-} \leq p^{+}<\infty$. If $\alpha+\frac{1}{k} \leq \frac{Q(x)}{p(x)}$ for some $k \geq 1$ and every $x \in X$, then

$$
\int_{B}\left[J_{\alpha}^{B} u(x)\right]^{p^{\#}(x)} d \mu(x) \leq C(C k)^{Q^{+} k}
$$


for every ball $B \subset X$ and every $u \in L^{p(\cdot)}(X)$ with $\|u\|_{L^{p(\cdot)(5 B)}} \leq 1$, where the constant $C$ does not depend on $k$.

Proof. Let $x \in B$ and let $0<\delta \leq 2 \operatorname{diam} B$ be a number to be specified later. Since $p$ is log-Hölder continuous, $p^{\prime}$ is too, and by Lemma 2.4 we obtain that

$$
\|1\|_{L^{p^{\prime}(\cdot)\left(B\left(x, 2^{i}\right)\right)}} \leq C \mu\left(B\left(x, 2^{i}\right)\right)^{\frac{1}{p^{\prime}(x)}} .
$$

Using Hölder's inequality for the first estimate, we have for every $x \in B$ that

$$
\begin{aligned}
\sum_{\delta \leq 2^{i} \leq 2 \operatorname{diam} B} & 2^{i \alpha} f_{B\left(x, 2^{i}\right)}|u(y)| d \mu(y) \\
& \lesssim \sum_{\delta \leq 2^{i}} \frac{2^{i \alpha}}{\mu\left(B\left(x, 2^{i}\right)\right)}\|u\|_{L^{p(\cdot)}\left(B\left(x, 2^{i}\right)\right)}\|1\|_{L^{p^{\prime}(\cdot)}\left(B\left(x, 2^{i}\right)\right)} \\
& \lesssim \sum_{\delta \leq 2^{i}} 2^{i \alpha} \mu\left(B\left(x, 2^{i}\right)\right)^{-1+1 / p^{\prime}(x)}=\sum_{\delta \leq 2^{i}} 2^{i \alpha} \mu\left(B\left(x, 2^{i}\right)\right)^{-1 / p(x)} \\
& \lesssim \sum_{\delta \leq 2^{i}} 2^{i \alpha-i \frac{Q(x)}{p(x)}} \leq \frac{\delta^{-Q(x) / p^{\#}(x)}}{1-2^{-Q(x) / p^{\#}(x)}} .
\end{aligned}
$$

In the last inequality we used the formula for the geometric sum. Since $Q(x) / p^{\#}(x) \geq 1 / k$ and $k \geq 1$ by assumption, we see that

$$
\left(1-2^{-Q(x) / p^{\#}(x)}\right)^{-1} \leq 2 k .
$$

Estimating a geometric sum by its first term, we find that

$$
\sum_{2^{i} \leq \delta} 2^{i \alpha} f_{B\left(x, 2^{i}\right)}|u(y)| d \mu(y) \lesssim \delta^{\alpha} \mathcal{M}\left(u \chi_{5 B}\right)(x) .
$$

Combining the estimates gives

$$
J_{\alpha}^{B} u(x) \lesssim \delta^{\alpha} \mathcal{M}\left(u \chi_{5 B}\right)(x)+k \delta^{-\frac{Q(x)}{p^{\#}(x)}} .
$$

For almost every $x$ we have $\mathcal{M}\left(u \chi_{5 B}\right)(x)<+\infty$, so by the previous estimate, the same is true for the Riesz potential.

If $\left[\mathcal{M}\left(u \chi_{5 B}\right)(x)\right]^{-\frac{p(x)}{Q(x)}}<2 \operatorname{diam}(B)$, we choose $\delta=\left[\mathcal{M}\left(u \chi_{5 B}\right)(x)\right]^{-\frac{p(x)}{Q(x)}}$. Then the previous inequality gives

$$
J_{\alpha}^{B} u(x) \lesssim k\left[\mathcal{M}\left(u \chi_{5 B}\right)(x)\right]^{1-\alpha \frac{p(x)}{Q(x)}} .
$$

If $\left[\mathcal{M}\left(u \chi_{5 B}\right)(x)\right]^{-\frac{p(x)}{Q(x)}} \geq 2 \operatorname{diam}(B)$, we choose $\delta=2 \operatorname{diam}(B)$. Now we have

$$
\delta^{\alpha} \leq\left[\mathcal{M}\left(u \chi_{5 B}\right)(x)\right]^{-\alpha \frac{p(x)}{Q(x)}}
$$

which by (4.6) implies (4.7). 
We raise both sides of (4.7) to the power of $p^{\#}(x)$ and integrate over $x \in B$. This yields

$$
\int_{B}\left[J_{\alpha}^{B} u(x)\right]^{p^{\#}(x)} d \mu(x) \leq(C k)^{Q^{+} k} \int_{X}\left[\mathcal{M}\left(u \chi_{5 B}\right)(x)\right]^{p(x)} d \mu(x) .
$$

By Theorem 4.2, the Hardy-Littlewood maximal operator is bounded and hence

$$
\left\|M u \chi_{5 B}\right\|_{L^{p(\cdot)}(X)} \lesssim\|u\|_{L^{p(\cdot)(5 B)}} \leq 1 .
$$

This implies that $\varrho_{L^{p(\cdot)}(X)}\left(M u \chi_{5 B}\right) \leq C$, which, combined with the previous inequality, gives

$$
\int_{X}\left[J_{\alpha}^{B} u(x)\right]^{p^{\#}(x)} d \mu(x) \leq C(C k)^{Q^{+k}} .
$$

Theorem 4.8. Let $\mu$ be lower Ahlfors $Q(\cdot)$-regular in a bounded set $X \subset \mathbb{R}^{n}$. Let $p$ be log-Hölder continuous in $X$ and let $1<p^{-} \leq p^{+}<\infty$. Then the following claims hold for every $u \in L^{p(\cdot)}(X)$.

(1) If $\alpha<\inf \frac{Q(x)}{p(x)}$, then for each ball $B \subset X$ we have

$$
\left\|J_{\alpha}^{B} u\right\|_{L^{p \#(\cdot)(B)}} \lesssim\|u\|_{L^{p(\cdot)(5 B)}} .
$$

(2) If $Q(x) \geq 1$ and $\alpha=\frac{Q(x)}{p(x)}$ for all $x \in X$, then there exists $C_{1}>1$ so that for each ball $B \subset X$ we have

$$
\int_{B} \exp \left(\frac{C_{1} J_{\alpha}^{B} u(x)}{\|u\|_{L^{p(\cdot)}(5 B)}}\right)^{\frac{1}{Q^{+}}} d \mu(x) \leq 2 .
$$

(3) If $\alpha>\sup \frac{Q(x)}{p(x)}$, then for each ball $B \subset X$ we have

$$
\left\|J_{\alpha}^{B} u\right\|_{L^{\infty}(B)} \lesssim\|u\|_{L^{p(\cdot)}(5 B)} .
$$

Proof. Using Proposition 4.4 for $u /\|u\|_{L^{p(\cdot)}(5 B)}$ and some sufficiently large $k$, we obtain claim (1).

Next we consider the case $\alpha=\frac{Q(x)}{p(x)}$. For every positive integer $k$ we define a function $\varepsilon_{k}: X \rightarrow(0, \infty)$ by $\varepsilon_{k}(x)=p(x)^{2} /\left(k / Q^{+}+p(x)\right)$. Then it follows that $\left(p-\varepsilon_{k}\right)^{\#}(x)=k / Q^{+}$. Since

$$
\|u\|_{L^{p(\cdot)-\varepsilon_{k}(\cdot)}(5 B)} \leq(1+\mu(5 B))\|u\|_{L^{p(\cdot)}(5 B)}
$$

we obtain by using Proposition 4.4 for the function $u /\|u\|_{L^{p(\cdot)(5 B)}}$ with the exponent $p-\varepsilon_{k}$ that

$$
\int_{B}\left(\frac{C_{1} J_{\alpha}^{B} u(x)}{\|u\|_{L^{p(\cdot)}(5 B)}}\right)^{\frac{k}{Q^{+}}} d \mu(x) \leq\left(C_{2} k\right)^{k},
$$

where $C_{1}$ is chosen so small that $C_{2}<1 / e$ (note that this can be done uniformly in $k$ ). The proposition is applicable, since $Q(x) \geq 1$ implies that 
$\alpha+\frac{1}{k} \leq \frac{Q(x)}{p(x)-\varepsilon_{k}(x)}$. Using a series expansion for $e^{x}$, we find that

$$
\begin{aligned}
\int_{B} \exp \left(\frac{C_{1} J_{\alpha}^{B} u(x)}{\|u\|_{L^{p(\cdot)}(5 B)}}\right)^{\frac{1}{Q^{+}}} d \mu(x) & =\sum_{k \geq 0} \frac{1}{k !} \int_{B}\left(\frac{C_{1} J_{\alpha}^{B} u(x)}{\|u\|_{L^{p(\cdot)}(5 B)}}\right)^{\frac{k}{Q^{+}}} d \mu(x) \\
& \leq \sum_{k \geq 0} \frac{\left(C_{2} k\right)^{k}}{k !}<\infty .
\end{aligned}
$$

Finally, we have the case $\alpha>\sup \frac{Q(x)}{p(x)}$. We denote $r=2 \operatorname{diam} B$. By Hölder's inequality and inequality (4.5) we obtain that

$$
\begin{aligned}
J_{\alpha}^{B} u(x) & =\sum_{2^{i} \leq r} 2^{i \alpha} f_{B\left(x, 2^{i}\right)}|u(y)| d \mu(y) \\
& \lesssim \sum_{2^{i} \leq r} 2^{i \alpha} \mu\left(B\left(x, 2^{i}\right)\right)^{-1+\frac{1}{p^{\prime}(x)}}\|u\|_{L^{p(\cdot)}\left(B\left(x, 2^{i}\right)\right)} \\
& \lesssim \sum_{2^{i} \leq r} 2^{i \alpha-i \frac{Q(x)}{p(x)}}\|u\|_{L^{p(\cdot)}\left(B\left(x, 2^{i}\right)\right)} \leq r^{\alpha-\sup \frac{Q(x)}{p(x)}}\|u\|_{L^{p(\cdot)}(B(x, r))} \\
& \leq\left(1+(\operatorname{diam} X)^{\alpha}\right)\|u\|_{L^{p(\cdot)}(5 B)} .
\end{aligned}
$$

Remark 4.9. There is a power $1 / Q^{+}$on the Riesz potential in (2), above. This exponent does not appear in the paper [14], but a careful scrutiny of their argument shows that in fact it should occur. In other words, their argument does not not prove their claim, only a weaker claim of the kind presented here.

Remark 4.10. We have stated the results in this section for metric measure spaces $(X, d, \mu)$ where $X \subset \mathbb{R}^{n}$ and the measure is lower Ahlfors regular. However, the condition $X \subset \mathbb{R}^{n}$ is needed only to imply that the maximal operator is bounded for constant exponents $p>1$. In particular, if our metric measure space is doubling or $(\varepsilon, M)$-directional, then we do not need to assume that $X \subset \mathbb{R}^{n}$.

\section{EMbeddings in HajŁasz SPACES}

We end this paper by applying the results of the previous section to obtain two embedding theorems for variable exponent and variable dimension.

In this section we assume that $\mu$ is lower Ahlfors $Q(\cdot)$-regular and doubling in a metric measure space $X$. Our basic example is the variable dimensional von Koch curve which does not contain rectifiable curves. Hence the definition of Sobolev spaces can not be based on upper gradients (which would give us Newtonian spaces). Instead of that we use the Hajłasz-Sobolev space $M^{1, p(\cdot)}$, which was introduced in [15].

A measurable function $u \in L^{p(\cdot)}(X)$ belongs to $M^{1, p(\cdot)}(X)$ if there exists a non-negative $g \in L^{p(\cdot)}(X)$ such that

$$
|u(x)-u(y)| \leq C d(x, y)(g(x)+g(y))
$$


for $\mu$-almost every $x, y \in X$. Any such function $g$ is called here a Hajłasz gradient of $u$. If $1<p^{-} \leq p^{+}<\infty$, then there exists a unique Hajłasz gradient $g$ which minimizes the norm in $M^{1, p(\cdot)}(X)$, [20, Proposition 3.1].

Sobolev embeddings. Our first embedding follows easily by combining the point-wise estimate (5.1) with the results of the previous section. Let $u \in M^{1, p(\cdot)}(X)$ and let $g \in L^{p(\cdot)}(X)$ be a Hajłasz gradient of $u$. Integrating both sides in (5.1) over $y$ and $x$ we obtain in a standard manner the Poincaré inequality

$$
\int_{B}\left|u(x)-u_{B}\right| d \mu \leq C(X) \operatorname{diam}(B) \int_{B} g(x) d \mu
$$

for every ball $B \subset X$. Since the measure is assumed to be doubling we find by $[14$, Theorem 5.2] that

$$
\left|u(x)-u_{B}\right| \leq C J_{1}^{B} g(x)
$$

for $\mu$-almost every $x \in B$. Now Theorem 4.8 yields the following result.

Corollary 5.4. Let $\mu$ be lower Ahlfors $Q(\cdot)$-regular and doubling in a bounded metric space $X$. Let $p$ be log-Hölder continuous in $X$ and let $1<p^{-} \leq p^{+}<$ $\infty$. If $1<\inf \frac{Q(x)}{p(x)}$, then for each ball $B \subset X$ we have

$$
\left\|u-u_{B}\right\|_{L^{p \#(\cdot)(B)}} \leq C\|g\|_{L^{p(\cdot)(5 B)}} .
$$

Trudinger-Sobolev embeddings. As the main result of this section we extend a Trudinger-Sobolev type embedding to our setting. Recall that in the classical case this embedding states that $W^{1, n}$ embeds into $\exp L^{n^{\prime}}$, where the exponent $n^{\prime}=n /(n-1)$ is the best possible. With variable exponent spaces we can prove a similar result for the case of variable dimensional spaces.

We will modify the proof of [14, Theorem 6.1] to the variable exponent, variable dimension case. We start with an auxiliary lemma.

Lemma 5.5. Let $p$ be bounded and $\log$-Hölder continuous, $B$ is a ball and $x \in \lambda B$, where $\lambda \geq 1$. If $\|u\|_{L^{p(\cdot)(B)}} \leq 1$, then

$$
f_{B} u d \mu \lesssim 1+\mu(B)^{s-\frac{1}{p(x)}}\left[\mathcal{M}\left(u^{p(\cdot)}\right)(x)\right]^{s} \varrho_{L^{p(\cdot)}(B)}(u)^{\frac{1}{p(x)}-s}
$$

for all $s<1 / p(x)$. Here the implicit constant depends on $\lambda$, $s$ and $p$. 
Proof. We start in the standard way, using Hölder's inequality for the constant exponent $p_{\lambda B}^{+}$:

$$
\begin{aligned}
\left(f_{B} u d \mu\right)^{p(x)} & \leq\left(f_{B} u^{p_{\lambda B}^{-}} d \mu\right)^{\frac{p(x)}{p_{\lambda B}^{-}}} \leq\left(f_{B} 1+u(y)^{p(y)} d \mu(y)\right)^{\frac{p(x)}{p_{\lambda B}^{-}}} \\
& \lesssim 1+\mu(B)^{-\frac{p(x)}{p_{\lambda B}^{-}}}\left(\int_{B} u(y)^{p(y)} d \mu(y)\right)^{\frac{p(x)}{p_{\lambda B}^{-}}} \\
& \leq 1+\mu(B)^{1-\frac{p(x)}{p_{\lambda B}^{-}}} f_{B} u(y)^{p(y)} d \mu(y),
\end{aligned}
$$

where we used that $\int u^{p} \leq 1$ in the last inequality. Since $p$ is log-Hölder continuous and $\mu$ is doubling, we find that

$$
\mu(B)^{1-\frac{p(x)}{p_{\lambda B}^{-}}} \lesssim\left(\mu(\lambda B)^{p_{\lambda B}^{-}-p(x)}\right)^{\frac{1}{p_{\lambda B}^{-}}} \leq C .
$$

Thus we have shown that

$$
f_{B} u d \mu \lesssim 1+\left(f_{B} u(y)^{p(y)} d \mu(y)\right)^{\frac{1}{p(x)}} .
$$

To conclude the proof we use that

$$
\begin{aligned}
& \left(f_{B} u(y)^{p(y)} d \mu(y)\right)^{\frac{1}{p(x)}} \\
& \quad=\mu(B)^{s-\frac{1}{p(x)}}\left(f_{B} u(y)^{p(y)} d \mu(y)\right)^{s}\left(\int_{B} u(y)^{p(y)} d \mu(y)\right)^{\frac{1}{p(x)}-s},
\end{aligned}
$$

and estimate the second factor in the last line by the maximal function.

Adapting [14, Section 6], we say that a metric measure space $X$ satisfies a chain condition if there is a constant $M$ such that for each $x \in X$ and all $0<R_{1}<R_{2}<\frac{1}{4}$ diam $X$ there is a finite sequence of balls $B_{0}, B_{1}, \ldots, B_{k}$ which satisfy

(1) $B_{0} \subset X \backslash B\left(x, R_{2}\right)$ and $B_{k} \subset B\left(x, R_{1}\right)$,

(2) $M^{-1} \operatorname{diam}\left(B_{i}\right) \leq \operatorname{dist}\left(x, B_{i}\right) \leq M \operatorname{diam}\left(B_{i}\right)$ for $i=0,1, \ldots, k$,

(3) $B_{i} \subset B\left(x, 4 R_{2}\right)$ for $i=0,1, \ldots, k$,

(4) there is a ball $B_{i}^{\prime} \subset B_{i} \cap B_{i+1}$ such that $B_{i} \cap B_{i+1} \subset M B_{i}^{\prime}$ for $i=$ $0,1, \ldots, k-1$ and

(5) no points of $X$ belongs to more than $M$ balls $B_{i}$.

It was shown in [14, Section 6] that if $X$ is a bounded connected doubling space then it satisfies this chain condition. Condition (3) is not explicitly stated in [14], but it can be easily deduced from the construction presented in [14], p. 30.

The following lemma is proved in the third paragraph of the proof of [14, Theorem 6.1].

Lemma 5.6. Let $r_{i}$ be the radii of the balls from a chain whose largest ball has radius $r$. Then $\sum r_{i}^{s} \lesssim r^{s} / s$ for any $s>0$. 
Theorem 5.7. Let $X$ be a bounded connected doubling space, and assume that $\mu$ is lower Ahlfors $Q(\cdot)$-regular, where $Q$ is log-Hölder continuous and $1<Q^{-} \leq Q^{+}<\infty$. Then there is a constant $C_{1}$, depending on $X$, such that

$$
f_{B} \exp \left(\frac{C_{1}\left|u(x)-u_{B}\right|}{\|g\|_{L^{Q(\cdot)}(5 B)}}\right)^{Q^{\prime}(x)} d \mu(x) \leq 2
$$

for every ball $B \subset X$ and for each $u \in M^{1, Q(\cdot)}(X)$ with Hajtasz gradient $g$.

Proof. Fix a ball $B \subset X$, $\operatorname{diam}(B) \leq \operatorname{diam}(X)$, with radius $r$ and a point $x \in B$. Let $u \in M^{1, p(\cdot)}(X)$ and assume without loss of generality that $\|g\|_{L^{Q(\cdot)(5 B)}}=1$. Let $B_{0}, \ldots, B_{k}$ be a chain associated to $x$ with $R_{2}=$ $\frac{1}{5} \operatorname{diam}(B)$ and $0<R_{1}<R_{2}$. By (1), (2) and (3) we have $\mu\left(B_{0}\right) \approx \mu(B)$ and by (3) $B_{i} \subset 5 B$ for every $i=0, \ldots, k$. By Lemma 6.2, [14], there exits an $R_{1}>0$ and a corresponding $k$ such that

$$
\left|u(x)-u_{B_{0}}\right| \lesssim \sum_{i=0}^{k} r_{i} f_{B_{i}} g d \mu .
$$

Let $q(x)=2 k Q(x) /(Q(x)-1)$ and note that $q$ is also log-Hölder continuous and satisfies $1<q^{-} \leq q^{+}<\infty$. We consider first the case $k>\left(Q^{+}-1\right) / 2$, and note that then $q>Q$. Let us denote $f(y)=g(y)^{Q(y)}$. By the chain property (2) we have $x \in(2 M+1) B_{i}$. Using Lemma 5.5 with $s=1 / q(x)$ and $\lambda=2 M+1$ in the previous inequality, we find that

$$
\begin{aligned}
\mid u(x) & -u_{B_{0}} \mid \lesssim \sum_{i=0}^{k} r_{i}\left[1+\mu\left(B_{i}\right)^{\frac{1}{q(x)}-\frac{1}{Q(x)}}(\mathcal{M} f(x))^{\frac{1}{q(x)}} \varrho_{L^{Q(\cdot)}\left(B_{i}\right)}(g)^{\frac{1}{Q(x)}-\frac{1}{q(x)}}\right] \\
& \lesssim \sum_{i=0}^{k}\left[r_{i}+r_{i}^{1-\frac{Q(x)}{q(x)^{2}}}\left(r_{i}^{\frac{Q(x)}{q(x)}} \mathcal{M} f(x)\right)^{\frac{1}{q(x)}}\left(\frac{\varrho_{L} Q(\cdot)\left(B_{i}\right)}{\mu(g)}\right)^{\frac{1}{Q(x)}-\frac{1}{q(x)}}\right] .
\end{aligned}
$$

The first sum on the right-hand-side is dominated by $r$, by Lemma 5.6. For the second term, notice that we regard $x$ as a fixed point, so all the exponents are actually non-variable. Hence the previous inequality is essentially the same as the one appearing in the proof of Theorem 6.1, [14], p. 31, lines 7-8. By replacing every $q$ by $q(x)$, every $s$ by $Q(x)$, and every $\varepsilon$ by $Q(x) / q(x)^{2}$ in paragraphs $2-4$ of the same proof, we arrive at the inequality

$$
\left|u(x)-u_{B_{0}}\right| \lesssim r+q(x)^{\frac{1}{q(x)}+\frac{1}{Q^{\prime}(x)}} \mu(B)^{\frac{1}{q(x)}-\frac{1}{Q(x)}} r[\mathcal{M} f(x)]^{\frac{1}{q(x)}},
$$

which corresponds to the estimate [14], p. 32, line 3. Since $\mu$ is doubling and $X$ is bounded, we obtain from the lower Ahlfors regularity that

$$
r^{Q(x)} \lesssim \mu(B(x, r)) \leq \mu(2 B) \lesssim \mu(B) .
$$

Hence we conclude that

$$
\left|u(x)-u_{B_{0}}\right| \lesssim \operatorname{diam}(X)+q(x)^{\frac{1}{q(x)}+\frac{1}{Q^{\prime}(x)}} \mu(B)^{\frac{1}{q(x)}}[\mathcal{M} f(x)]^{\frac{1}{q(x)}} .
$$

Next, we use the estimate $\left|u(x)-u_{B}\right| \leq\left|u(x)-u_{B_{0}}\right|+\left|u_{B_{0}}-u_{B}\right|$. By the Poincaré inequality, Hölder's inequality, Lemma 2.4, the assumption 
$\|g\|_{L^{Q(\cdot)(5 B)}}=1$ and lower Ahlfors regularity we may estimate the second term as follows:

$$
\begin{aligned}
\left|u_{B_{0}}-u_{B}\right| & \leq\left|u_{B_{0}}-u_{5 B}\right|+\left|u_{B}-u_{5 B}\right| \leq f_{B_{0}}\left|u-u_{5 B}\right| d \mu+f_{B}\left|u-u_{5 B}\right| d \mu \\
& \lesssim f_{5 B}\left|u-u_{5 B}\right| d \mu+f_{5 B}\left|u-u_{5 B}\right| d \mu \lesssim r f_{5 B} g d \mu \\
& \lesssim \frac{r}{\mu(5 B)}\|1\|_{L^{Q^{\prime}(\cdot)(5 B)}}\|g\|_{L^{Q(\cdot)}(5 B)} \lesssim \frac{r}{\mu(B)} \mu(B)^{\frac{1}{Q^{\prime}(x)}} \leq C .
\end{aligned}
$$

We combine this with (5.8) and conclude that

$$
\left|u(x)-u_{B}\right| \lesssim 1+q(x)^{\frac{1}{q(x)}+\frac{1}{Q^{\prime}(x)}} \mu(B)^{\frac{1}{q(x)}}[\mathcal{M} f(x)]^{\frac{1}{q(x)}} .
$$

We raise both sides of this inequality to the power $q(x) / 2$ and integrate over $x \in B$. This gives

$$
\begin{aligned}
& \int_{B}\left|u(x)-u_{B}\right|^{\frac{q(x)}{2}} d \mu(x) \\
& \quad \lesssim C^{q_{B}^{+}} \mu(B)+C^{q_{B}^{+}} \int_{B} q(x)^{\frac{1}{2}+\frac{q(x)}{2 Q^{\prime}(x)}}[\mu(B) \mathcal{M} f(x)]^{\frac{1}{2}} d \mu(x) .
\end{aligned}
$$

Next we use the expression $q(x)=2 k Q^{\prime}(x) \leq 2 k\left(Q_{B}^{-}\right)^{\prime}$ for $q$, and note that $2\left(Q_{B}^{-}\right)^{\prime}$ is bounded by a constant. Thus our inequality becomes

$$
\int_{B}\left|u(x)-u_{B}\right|^{k Q^{\prime}(x)} d \mu(x) \lesssim C^{k} \mu(B)+C^{k} k^{\frac{1}{2}+k} \mu(B)^{\frac{1}{2}} \int_{B}[\mathcal{M} f]^{\frac{1}{2}} d \mu .
$$

Using the weak type estimate (4.1) it follows by [14, Theorem 14.11] that

$$
\int_{B}[\mathcal{M} f]^{\frac{1}{2}} d \mu \lesssim\left(\mu(B) \int_{5 B} f d \mu\right)^{\frac{1}{2}} .
$$

So we continue our previous estimate by

$$
\begin{aligned}
\int_{B}\left|u(x)-u_{B}\right|^{k Q^{\prime}(x)} d \mu(x) & \lesssim C^{k} \mu(B)+C^{k} k^{\frac{1}{2}+k} \mu(B)\left(\int_{5 B} f d \mu\right)^{\frac{1}{2}} \\
& \leq \mu(B) C^{k}\left(1+k^{\frac{1}{2}+k}\right) .
\end{aligned}
$$

We divide the previous inequality by $\mu(B)$ and obtain

$$
f_{B}\left|u(x)-u_{B}\right|^{k Q^{\prime}(x)} d \mu(x) \lesssim C^{k}\left(1+k^{\frac{1}{2}+k}\right) .
$$

Recall that this was derived under the assumption $k>\left(Q^{+}-1\right) / 2$.

Let us then consider the case $0<k \leq\left(Q^{+}-1\right) / 2$. By [25], Theorem 2.8, we obtain

$$
\|g\|_{L^{\frac{k Q(\cdot)}{Q(\cdot)-1+k}(5 B)}} \leq(1+\mu(5 B))\|g\|_{L^{Q(\cdot)}(5 B)}=1+\mu(5 B) .
$$

Applying (5.3) for the functions $\frac{u}{(1+\mu(5 B))}$ and $\frac{g}{(1+\mu(5 B))}$, we have

$$
f_{B}\left|u(x)-u_{B}\right|^{k Q^{\prime}(x)} d \mu(x) \lesssim(1+\mu(5 B))^{\left(\frac{Q^{+}-1}{2}\right)\left(Q^{\prime}\right)^{+}} f_{B} J_{1}\left(\frac{g(x)}{(1+\mu(5 B)}\right)^{k Q^{\prime}(x)} d \mu .
$$


The integral average on the right hand side can be estimated with the aid of Proposition 4.4 by choosing

$$
p(x):=\frac{k Q(x) Q^{\prime}(x)}{k Q^{\prime}(x)+Q(x)} \quad \text { and } \quad k^{*} \geq \frac{\left(Q^{+}-1\right)\left(Q^{\prime}\right)^{+}}{2 Q^{-}}
$$

since then $p^{\#}(x)=k Q^{\prime}(x)$ and $k^{*}$ satisfies $1+\frac{1}{k^{*}} \leq \frac{Q(x)}{p(x)}$ for all $x \in X$. By Proposition 4.4,

$$
f_{B} J_{1}\left(\frac{g(x)}{(1+\mu(5 B)}\right)^{k Q^{\prime}(x)} d \mu \lesssim\left(C k^{*}\right)^{Q^{+} k^{*}} \leq C,
$$

so (5.9) holds in this case also.

Let $t \in(0,1)$. Using a series expansion for the exponential function and the estimate (5.9), we find that

$$
\begin{gathered}
f_{B} \exp \left[\left(t\left|u(x)-u_{B}\right|\right)^{Q^{\prime}(x)}\right] d \mu(x)=\sum_{k=0}^{\infty} f_{B} \frac{1}{k !}\left[\left(t\left|u(x)-u_{B}\right|\right)^{k Q^{\prime}(x)}\right] d \mu(x) \\
\leq \sum_{k=0}^{\infty} \frac{t^{k\left(Q_{B}^{+}\right)^{\prime}}}{k !} f_{B}\left|u(x)-u_{B}\right|^{k Q^{\prime}(x)} d \mu(x) \lesssim 1+\sum_{k=1}^{\infty} \frac{\left(C t^{\left(Q_{B}^{+}\right)^{\prime}}\right)^{k}}{k !} k^{1+k} .
\end{gathered}
$$

By the ratio test, we see that the sum is convergent if $t<[e / C]^{-1 /\left(Q_{B}^{+}\right)^{\prime}}$. Thus $t$ can be chosen so small that the upper bound is 2 , which completes the proof.

\section{Acknowledgment}

We would like to thank T. Shimomura for comments on this manuscript.

\section{REFERENCES}

[1] E. Acerbi and G. Mingione: Gradient estimates for the $p(x)$-Laplacean system, $J$. Reine Angew. Math., to appear.

[2] D. Avnir, D. Farin and P. Pfeifer: Molecular Fractal Surfaces, Nature 308 (1984), 261-263.

[3] D. Cruz-Uribe, A. Fiorenze, J.M. Martell and C. Pérez: The boundedness of classical operators on variable $L^{p}$ spaces, Ann. Acad. Sci. Fenn. Math., to appear.

[4] L. Diening: Maximal function on generalized Lebesgue spaces $L^{p(\cdot)}$, Math. Inequal. Appl. 7 (2004), no. 2, 245-254.

[5] L. Diening: Riesz potential and Sobolev embeddings of generalized Lebesgue and Sobolev spaces $L^{p(\cdot)}$ and $W^{k, p(\cdot)}$, Math. Nachr. 263 (2004), no. 1, 31-43.

[6] L. Diening and M. Růžička: Calderón-Zygmund operators on generalized Lebesgue spaces $L^{p(\cdot)}$ and problems related to fluid dynamics, J. Reine Angew. Math. 563 (2003), 197-220.

[7] K. Falconer: Techniques in fractal geometry, John Wiley \& Sons, Ltd., Chichester, 1997.

[8] K. Falconer and T. O'Neil: Convolutions and the geometry of multifractal measures. Math. Nachr. 204 (1999), 61-82.

[9] D. Farin, A. Volpert and D. Avnir: Determination of Adsorption Conformation from Surface Resolution Analysis, J. Am. Chem. Soc. 107 (1985), 3368-3370. 
[10] J. Feder and A. Aharony (eds.): Fractals in Physics, Proceedings of the International Conference honoring Benoit B. Mandelbrot on his 65th birthday (Vence, 1989), NorthHolland Publishing Co., Amsterdam, 1990. [Reprint of Phys. D 38 (1989), no. 1-3.]

[11] H. Federer: Geometric Measure Theory, Springer-Verlag, Berlin-Heidelberg-New York, 1969.

[12] T. Futamura, Y. Mizuta and T. Shimomura: Sobolev embeddings for variable exponent Riesz potentials on metric spaces, preprint (2004).

[13] P. Hajłasz: Sobolev spaces on an arbitrary metric spaces, Potential Anal. 5 (1996), 403-415.

[14] P. Hajłasz and P. Koskela: Sobolev met Poincaré, Mem. Amer. Math. Soc. 145 (2000), no. 688.

[15] B. Hambly: Brownian motion on a random recursive Sierpinski gasket, Ann. Probab. 25 (1997), no. 3, 1059-1102.

[16] B. Hambly and T. Kumagai: Diffusion processes on fractal fields: heat kernel estimates and large deviations, Probab. Theory Related Fields 127 (2003), no. 3, 305-352.

[17] P. Harjulehto and P. Hästö: Lebesgue points in variable exponent spaces, Ann. Acad. Sci. Fenn. Math. 29 (2004), 295-306.

[18] P. Harjulehto, P. Hästö and V. Latvala: Lebesgue points in variable exponent Sobolev spaces on metric measure spaces, Complex Analysis and Free Boundary Flows, Transactions of the Institute of Mathematics of the National Academy of Sciences of Ukraine, 2004, vol. 1, no. 3, 87-99.

[19] P. Harjulehto, P. Hästö and M. Pere: Variable exponent Lebesgue spaces on metric spaces: the Hardy-Littlewood maximal operator, Real Anal. Exchange 30 (2004/2005), no. 1, 87-104.

[20] P. Harjulehto, P. Hästö and M. Pere: Variable exponent Sobolev spaces on metric measure spaces, preprint (2004).

[21] J. Heinonen: Lectures on Analysis on Metric Spaces, Springer-Verlag, New York, 2001.

[22] E. Järvenpää, M. Järvenpää and M. Llorente: Local dimensions of sliced measures and stability of packing dimensions of sections of sets, Adv. Math. 183 (2004), no. 1, 127-154.

[23] V. Kokilashvili and S. Samko: On Sobolev theorem for Riesz type potentials in the Lebesgue spaces with variable exponent, Z. Anal. Anwendungen 22 (2003), no. 4, 899-910.

[24] V. Kokilashvili and S. Samko: Maximal and fractional operators in weighted $L^{p(x)}$ spaces, Rev. Mat. Iberoamericana 20 (2004), no. 2, 493-515.

[25] O. Kováčik and J. Rákosník: On spaces $L^{p(x)}$ and $W^{1, p(x)}$, Czechoslovak Math. J. 41(116) (1991), 592-618.

[26] P. Mattila: Geometry of Sets and Measures in Euclidean Spaces, Cambridge University Press, Cambridge, 1995.

[27] Y. Mizuta and T. Shimomura: Continuity of Sobolev functions of variable exponent on metric spaces, Proc. Japan Acad. Ser. A Math. Sci. 80 (2004), no. 6, 96-99.

[28] Y. Mizuta and T. Shimomura: Sobolev's inequality for Riesz potentials with variable exponent satisfying a log-Hölder condition at infinity, J. Math. Anal. Appl., to appear.

[29] H. Takayasu: Fractals in the Physical Sciences, Manchester University Press, Manchester-New York, 1990

P. Harjulehto and P. Hästö

Department of Mathematics and Statistics

P.O. Box 68

FIN-00014 UNIVERSITY OF HELSINKI

FINLAND 
V. Latvala

Department of Mathematics

UNIVERSITY OF JOENSUU

P.O. Box 111

Fin-80101 JoensuU

FinlAND

Research group home-page:

http://mathstat.helsinki.fi/analysis/varsobgroup/

E-mail addresses:

petteri.harjulehto@helsinki.fi

peter.hasto@helsinki.fi

visa.latvala@joensuu.fi 